\title{
Cyclic Networks of Quantum Gates
}

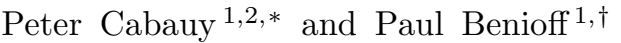 \\ ${ }^{1}$ Physics Division, Argonne National Laboratory, Argonne, IL 60439 and \\ ${ }^{2}$ Applied Physics Department, University of Michigan, Ann Arbor, MI 48109
}

(Dated: November 5, 2018)

\begin{abstract}
In this article initial steps in an analysis of cyclic networks of quantum logic gates will be given. Cyclic networks are those in which the qubit lines are loops. In our investigations of cyclic networks of quantum gates we have studied one and two qubit systems plus two qubit systems connected to another qubit on an acyclic line. The analysis includes: classifying networks into groups, the dynamics of the qubits in the cyclic network, and the perturbation effects of an acyclic qubit acting on a cyclic network of quantum gates. This will be followed by a discussion on quantum algorithms and quantum information processing with cyclic networks of quantum gates, a novel implementation of a cyclic network quantum memory and quantum sensors via cyclic networks will also be discussed.
\end{abstract}

PACS numbers: 03.67.-a, 03.67.Lx, 84.35.+i

\section{INTRODUCTION}

To date, quantum information research has largely been concerned with processing information in an acyclic manner. This is due to the fact that in 1993 Andrew Yao [1] showed that any function computable in polynomial time by a quantum Turing machine can also be computed by a polynomial-sized, acyclic quantum gate array. This result showed that acyclic quantum gate arrays are sufficient for modelling computations and consequently in the years after Yao's result, quantum algorithms have been described based on acyclic arrays.

Interestingly, some algorithms have a cyclic iterative component built into their evolution yet they are still expressed by acyclic arrays. For instance, Grover's search algorithm, expressed as an acyclic array, repeats a set of quantum gate operations $\mathrm{O}(\sqrt{n})$ times to reach a solution [2]. These repeating set of gates can just as easily be expressed as a single set of quantum gates looped back onto it-self where the qubits are measured after $\mathrm{O}(\sqrt{n})$ cycles around the network. In other words, the acyclic array for Grover's algorithm can be depicted more compactly with a cyclic network. Another iterative process that can easily be expressed as a cyclic network is quantum phase estimation [3, 4. In this algorithm, typically represented by an acyclic array, a unitary operator is iterated conditionally for $\mathrm{O}\left(2^{t}\right)$ times (where $t$ is chosen to be the desired bit length estimate of the phase). This iterated operator can also be expressed as a cyclic network in conjunction with an acyclic line acting conditionally on the cyclic network (to be shown in Section 4, Figure 9).

In realizing that these quantum algorithms have an iterative property; yet are typically depicted by acyclic gate arrays, it becomes interesting to study the structure and evolution of cyclic networks of quantum gates. One

\footnotetext{
*Electronic address: cabauv@umich.edu
}

${ }^{\dagger}$ Electronic address: pbenioff@anl.gov reason for this interest is that cyclic networks are common place in "classical" computing. For instance, the "do loop" subroutine is often used in programs and would be cumbersome to implement using acyclic methods. Also, computer hardware is comprised of wiring circuitry which involve many loops.

Other reasons for studying cyclic networks of quantum gates stem from the fact that some physical systems may require networks that are compact with just a few inputs and outputs, and a great deal of internal looping. For example, one can imagine a quantum robot [5, 6] moving about an environment (i.e. a lattice) where an on-board quantum computer controls the robot's operations. In this case, it is difficult to describe the on board quantum computer with acyclic arrays given the limited volume that a quantum robot encompasses.

Another reason for studying cyclic networks is that, unlike the case for acyclic networks, they are not limited to computations that halt. Halting computations can be carried out in cyclic networks by periodic measurements of a flag qubit to determine if the computation has halted. Also it may not even be decidable which acyclic array is equivalent to a cyclic network of arbitrary complexity, or even if an equivalent acyclic array exists. This is based on the observation that the existence problem seems equivalent to the unsolvable halting problem for Turing machines.

At the present time some research into the "cyclic" processing of quantum information within the context of feedback systems has been looked at by Lloyd [7]. In his investigation, it was shown that quantum systems benefit from information in a feedback procedure where no measurement is made. Rather, the information is processed by a "quantum governor" that may be modelled by a quantum gate network; which in turn sends quantum feedback information back to the quantum system, thus completing a cyclic quantum gate procedure. Other investigations in quantum feedback within the context of quantum control have also appeared in the literature [8, 9, 10].

It is possible that an outright investigation into cyclic 


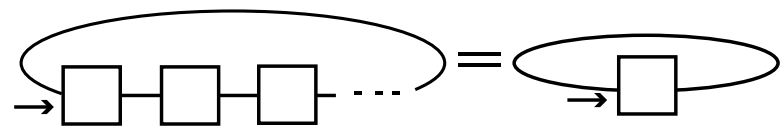

(a)

(b)

FIG. 1: (a)-A sequence of single qubit gates where each gate belongs to the $\mathrm{U}(2)$ group but the parameters of each gate may vary from gate to gate. (b)-One gate with appropriate parameters in $\mathrm{U}(2)$ can simulate the cyclic network on the left hand side.

networks of quantum gates may inspire new methods for designing quantum algorithms and overall quantum information processing. In our investigations of cyclic networks, we have studied simple one and two qubit systems. Although the one qubit cyclic networks are quite trivial, they will be used to motivate the analysis of the less trivial two qubit cyclic networks. In this article, cyclic networks will be classified into groups (Section II), the dynamics of the qubits moving around the cyclic networks will be examined (Section III), and the perturbation effects of an acyclic qubit acting on a cyclic network will be looked at (Section IV). A discussion will follow on how cyclic networks might be used in quantum algorithms and quantum information processing, a description of a novel implementation of a cyclic network quantum memory will be given, and quantum sensors via cyclic networks will also be discussed.

\section{STRUCTURE OF SIMPLE CYCLIC NETWORKS}

In order to explore simple cyclic networks it is useful to classify them according to the structure of the unitary matrix representing the cyclic network's combined gateoperation for one iteration of the qubit(s). It is helpful to use the following two conventions when classifying these networks into groups: The first convention sets the number of qubits per line to only one. The second convention requires the qubits to move in the direction of the arrows (see Figures 1, 2) and to move simultaneously through both lines of any two qubit gate.

As will become evident later, one reason for classifying these cyclic networks (restricted to the above two conventions) is to be able to compactly express complicated gate arrangements within a cyclic network with fewer gates. This will allow for an easy way to understand the evolution of the qubit's wavefunction for complicated gate arrangements by using a less complicated arrangement that represents its group structure.

\section{A. One Qubit Structure}

As a simple introductory example to the classification process, consider the one qubit cyclic network in Figure 1(a). A cyclic network of this type may be simulated by a single qubit gate acting on the $|0\rangle,|1\rangle$ basis with the general $\mathrm{U}(2)$ group (Figure $1(\mathrm{~b})$ ). In matrix form the well known U(2) group [11 may be represented by

$$
e^{i \delta}\left(\begin{array}{cc}
e^{i \alpha} \cos \phi & e^{i \beta} \sin \phi \\
-e^{-i \beta} \sin \phi & e^{-i \alpha} \cos \phi
\end{array}\right)
$$

The cyclic network may be classified further if the single qubit gate in Figure 1(b) is reduced to the general $\mathrm{SU}(2)$ group by setting $\delta=0$. In this case, the gates represented in Figure 1(a) are restricted to $\mathrm{SU}(2)$ and its subgroups. Similarly, if the single qubit gate is reduced to the $\mathrm{SO}(2)$ group where $\alpha=\beta=\delta=0$, then the gates in Figure 1(a) are restricted to the $\mathrm{SO}(2)$ group.

\section{B. Two Qubit Structure}

Gates with two interacting qubits have a more interesting structure and can be similarly classified into $\mathrm{U}(2)$, $\mathrm{SU}(2)$ and $\mathrm{SO}(2)$ groups. (For an overview of quantum gates see reference [12, 13, 14, 15].) The action of a general Control-U(2) gates may be defined as

$$
G_{d n}(\alpha, \phi, \beta, \delta)=\left(\begin{array}{cccc}
1 & 0 & 0 & 0 \\
0 & 1 & 0 & 0 \\
0 & 0 & e^{i(\alpha+\delta)} \cos \phi & e^{i(\beta+\delta)} \sin \phi \\
0 & 0 & -e^{-i(\beta-\delta)} \sin \phi & e^{i(-\alpha+\delta)} \cos \phi
\end{array}\right)
$$

where the $\mathrm{U}(2)$ matrix acts on the $|10\rangle,|11\rangle$ basis, and

$$
G_{u p}(\alpha, \phi, \beta, \delta)=\left(\begin{array}{cccc}
1 & 0 & 0 & 0 \\
0 & e^{i(\alpha+\delta)} \cos \phi & 0 & e^{i(\beta+\delta)} \sin \phi \\
0 & 0 & 1 & 0 \\
0 & -e^{-i(\beta-\delta)} \sin \phi & 0 & e^{i(-\alpha+\delta)} \cos \phi
\end{array}\right)
$$

where the $\mathrm{U}(2)$ matrix acts on $|01\rangle,|11\rangle$ basis. (Note: schematically $G_{d n}$ is represented by $\overline{-\square}$ and $G_{u p}$ is represented by - $\square$ ) Both control gates act on a column vector representing the binary basis in lexicographical order.

In considering a product of gates $G_{d n}$ in Figure 2(a) the product of $\mathrm{U}(2)$ matrices only acts on the lower $2 \times 2$ matrix of $G_{d n}$. Therefore, the consecutive product of similarly oriented $G_{d n}$ gates is equivalent to just one $G_{d n}$ gate as in Figure 2(b). (Note, the network has the same group properties as in the single qubit case and may again be restricted to the $\mathrm{SU}(2)$ and $\mathrm{SO}(2)$ groups.) This analysis is similarly true for the $G_{u p}$ gates with the exception that the $\mathrm{U}(2)$ portion of the $G_{u p}$ matrix acts on the basis states $|01\rangle,|11\rangle$ rather than $|10\rangle,|11\rangle$ for the $G_{d n}$ case.

The ability to compress similarly oriented control gates as in Figure 2(a) to just one control gate as in Figure 2(b) raises the question of how many control gates are needed to simulate any arrangement of alternating gates as in Figure 2(c). The answer to this question can be found by looking at the matrix structure of the alternating product 


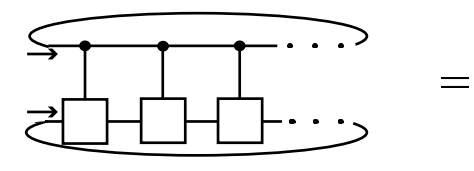

(a)

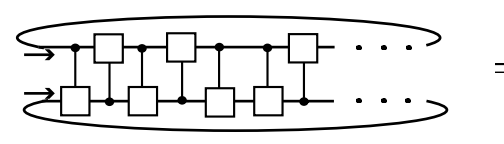

(c)

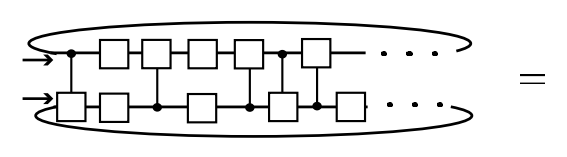

(e) (b)

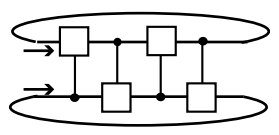

(d)

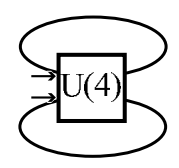

(f)

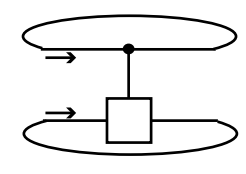

FIG. 2: (a,c,e)-A sequence of gates making U(2) transformations on a two qubit cyclic network. Note, each gate may be a different $\mathrm{U}(2)$ transformation with different parameters in $\mathrm{U}(2)$. (b,d,f)-A sequence of gates that can simulate the cyclic network on the left hand side with fewer gates.

of matrices $G_{u p}$ and $G_{d n}$ represented by

$$
G=\left(\begin{array}{cccc}
1 & 0 & 0 & 0 \\
0 & m_{11} & m_{12} & m_{13} \\
0 & m_{21} & m_{22} & m_{23} \\
0 & m_{31} & m_{32} & m_{33}
\end{array}\right)
$$

The product of assorted $G_{u p}$ and $G_{d n}$ control gate operations "actively" operate on three basis states $|01\rangle,|10\rangle,|11\rangle$ with the lower $3 \times 3$ matrix which may be called M. In contrast, the basis state $|00\rangle$ is only multiplied by the identity operation.

Since the active operations are limited to the lower $3 \times 3$ matrix $\mathrm{M}$, it becomes evident that the most general $3 \times 3$ unitary matrix $\mathrm{U}(3)$ will encompass all sequences of $G_{u p}$ and $G_{d n}$ gate operations. This matrix will be fairly complicated with nine parameters making it difficult to find an arrangement of control gates that will give $M$ as a general $\mathrm{U}(3)$ matrix.

Fortunately, a prescription for a general U(3) matrix expressed as a product of $\mathrm{U}(2)$ transformations exists 16, 17. This approach considerably simplifies the process of finding the control gate arrangement representing a general $\mathrm{U}(3)$ operation because control gates are also $\mathrm{U}(2)$ transformations.

Following the prescription given in reference [17 the general U(3) matrix may be written as

$$
D\left(1, \gamma_{1}, \gamma_{2}, \gamma_{3}\right) U_{3,4}\left(\phi_{1}, \beta_{1}\right) U_{2,3}\left(\phi_{2}, \beta_{2}\right) U_{2,4}\left(\phi_{3}, \beta_{3}\right)
$$

where $D\left(1, \gamma_{1}, \gamma_{2}, \gamma_{3}\right)$ represents a diagonal matrix with corresponding matrix entries $1, e^{i \gamma_{1}}, e^{i \gamma_{2}}, e^{i \gamma_{3}}$. The $U_{p, r}(\phi, \beta)$ matrix elements are obtained from a four dimensional identity matrix with elements $U_{p, p}, U_{r, p}, U_{p, r}$ and $U_{r, r}$ replaced with the corresponding unitary matrix elements in Equation 1 with $\alpha, \delta=0$. The mapping of the elements is as follows:

$$
\begin{aligned}
U_{3,4}(\phi, \beta) & =\left(\begin{array}{cccc}
1 & 0 & 0 & 0 \\
0 & 1 & 0 & 0 \\
0 & 0 & \cos \phi & e^{i \beta} \sin \phi \\
0 & 0 & -e^{-i \beta} \sin \phi & \cos \phi
\end{array}\right) \\
U_{2,3}(\phi, \beta) & =\left(\begin{array}{cccc}
1 & 0 & 0 & 0 \\
0 & \cos \phi & e^{i \beta} \sin \phi & 0 \\
0 & -e^{-i \beta} \sin \phi & \cos \phi & 0 \\
0 & 0 & 0 & 1
\end{array}\right) \\
U_{2,4}(\phi, \beta) & =\left(\begin{array}{cccc}
1 & 0 & 0 & 0 \\
0 & \cos \phi & 0 & e^{i \beta} \sin \phi \\
0 & 0 & 1 & 0 \\
0 & -e^{-i \beta} \sin \phi & 0 & \cos \phi
\end{array}\right)
\end{aligned}
$$

(Note, that in this notation the general $\mathrm{U}(3)$ matrix appears as the lower $3 \times 3$ matrix in order to conform with the typical matrix structure for a product of $G_{u p}$ and $G_{d n}$ gates as in Equation 1.)

A quick inspection of $U_{3,4}$ and $U_{2,4}$ matrices shows us that these are nothing more than control gates $G_{u p}$ and $G_{d n}$ respectively. However, $U_{23}$ does not have this form but may be converted into gate form by realizing that

$$
U_{2,3}\left(\phi_{2}, \beta_{2}\right)=U_{24}\left(-\frac{\pi}{2}, 0\right) U_{3,4}\left(\phi_{2},-\beta_{2}\right) U_{24}\left(\frac{\pi}{2}, 0\right)
$$

Therefore, by including Equation 9 into Equation 5 , distributing the diagonal elements of $D\left(1, \gamma_{1}, \gamma_{2}, \gamma_{3}\right)$ and reducing similarly oriented control gates to just one control gate, the $\mathrm{U}(3)$ matrix is reduced to the following form of $G_{u p}$ and $G_{d n}$ gates:

$$
\begin{aligned}
& U_{3,4}\left(\phi_{1}, \beta_{1}, \gamma_{2}, \gamma_{3}\right) U_{2,4}\left(-\frac{\pi}{2}, 0, \gamma_{1}, 0\right) \\
& \cdot U_{3,4}\left(\phi_{2},-\beta_{2}, 0,0\right) U_{2,4}\left(\phi_{3}+\frac{\pi}{2}, \beta_{3},-\beta_{3}, \beta_{3}\right)
\end{aligned}
$$

Note, the $U_{p, r}$ matrices have been extended to have two more variables where

$$
U_{2,4}\left(\phi, \beta, \gamma^{\prime}, \gamma^{\prime \prime}\right)=D\left(1, \gamma^{\prime}, 1, \gamma^{\prime \prime}\right) U_{2,4}(\phi, \beta, 0,0)
$$

and

$$
U_{3,4}\left(\phi, \beta, \gamma^{\prime}, \gamma^{\prime \prime}\right)=D\left(1,1, \gamma^{\prime}, \gamma^{\prime \prime}\right) U_{3,4}(\phi, \beta, 0,0)
$$

where $U_{p, r}(\phi, \beta)=U_{p, r}(\phi, \beta, 0,0)$.

Using this arrangement of matrices to give a general $\mathrm{U}(3)$ operation with four alternating control gates, answers the question of how many control gates are needed to simulate any number or sequence of control gates. This can be represented pictorially as in Figure 2(d).

The question arises regarding how many control gates are needed to simulate any alternating arrangement of Control-SU(2) and Control-SO(2) gates with at least one 
Control-SU(2) gate present. It is seen that four control gates is sufficient. This is due to the fact that an arrangement of this type will only restrict the matrix $M$ to an $\mathrm{SU}(3)$ matrix which is the same as requiring

$$
\delta_{1}+\delta_{2}+\delta_{3}=0
$$

This on its own is not sufficient to reduce the number of control gates.

On the other hand, if the cyclic network in Figure 2(c) is limited to only Control-SO(2) gates, then the number of gates required to simulate any arrangement is three. In fact, there is a very simple visual interpretation for this result. Consider the 3 basis states that M operates on $|01\rangle,|10\rangle,|11\rangle$ to be the 3 dimensional cartesian axes (x,y,z). An application $G_{d n}(\phi)$ on the two qubits leaves the coefficient of the basis state $|00\rangle$ unchanged. However, it rotates a vector representing the qubit-wavefunction about the $|01\rangle$ state (x-axis). Similarly, an application of $G_{u p}(\phi)$ is nothing more than a rotation about the $|10\rangle$ state (y-axis). Given this, it is well known that 3 rotations about alternating axes (i.e $\mathrm{x}$ and $\mathrm{y}$ axis) give any rotation in 3 dimensional space or the $\mathrm{SO}(3)$ group [18]. Therefore, an application of three alternating gates such as

$$
\begin{aligned}
& G_{u p}\left(\phi_{1}\right) G_{d n}\left(\phi_{2}\right) G_{u p}\left(\phi_{3}\right)=R_{y}\left(\phi_{1}\right) R_{x}\left(\phi_{2}\right) R_{y}\left(\phi_{3}\right) \\
& G_{d n}\left(\phi_{1}\right) G_{u p}\left(\phi_{2}\right) G_{d n}\left(\phi_{3}\right)=R_{x}\left(\phi_{1}\right) R_{y}\left(\phi_{2}\right) R_{x}\left(\phi_{3}\right)
\end{aligned}
$$

gives a general operation in $\mathrm{SO}(3)$, ultimately showing that three alternating gates is sufficient to simulate any number or arrangement of Control-SO(2) gates.

Up to this point, the two qubit cyclic networks have been classified into groups varying from $\mathrm{U}(3)$ through $\mathrm{SO}(2)$ control gate arrangements. This however does not encompass the most general two qubit network where single qubit gates are used along with control gates. The most general two qubit network is the U(4) group and it can encompasses any arrangement of single qubit gates and two qubit control gates as depicted in Figure 2 (e).

A general U(4) matrix represented by a product of $\mathrm{U}(2)$ transformations can again be found in reference 17] and is given by

$$
\begin{aligned}
& D\left(\gamma_{1}, \gamma_{2}, \gamma_{3}, \gamma_{4}\right) U_{34}\left(\phi_{1}, \theta_{1}\right) \\
& \cdot U_{23}\left(\phi_{2}, \theta_{2}\right) U_{24}\left(\phi_{3}, \theta_{3}\right) U_{12}\left(\phi_{4}, \theta_{4}\right) \\
& \cdot U_{13}\left(\phi_{5}, \theta_{5}\right) U_{14}\left(\phi_{6}, \theta_{6}\right)
\end{aligned}
$$

Each of these matrices can be converted into gates as follows (Note, the labelling of the boxes denote the type of $\mathrm{U}(2)$ operation to be implemented by either a single qubit gate or "conditionally" by a control gate.)

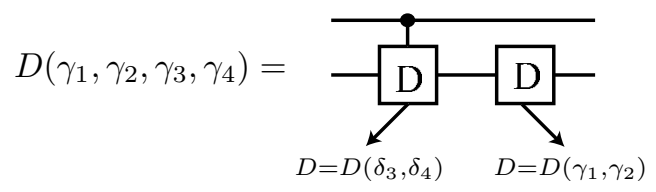

where $\gamma_{1}+\delta_{3}=\gamma_{3}$ and $\gamma_{2}+\delta_{4}=\gamma_{4}$.
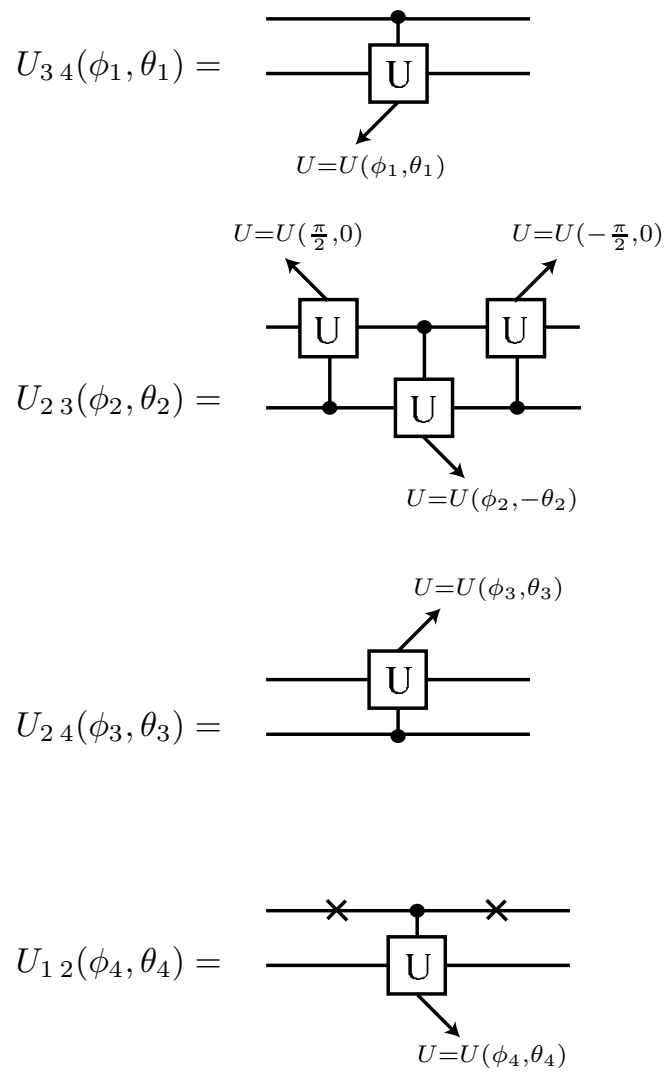

The $\times$ represents the Not operation,
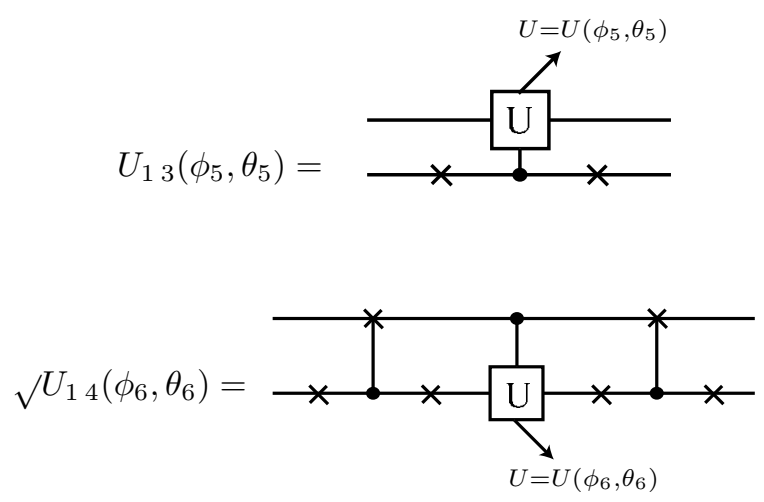

and the two qubit gate with the $\mathbf{x}$ is the Control-Not gate.

Therefore, by connecting these gates end to end (in reverse order due to the direction of the cycling qubits) a general $\mathrm{U}(4)$ cyclic network is achieved and may be represented by the two qubit $U(4)$ box in Figure 2(f). Furthermore, by using the grouping of single qubit gates and control gates discussed thus far, it is possible to reduce the number of gates in the $\mathrm{U}(4)$ network to just eight control gates and five single qubit gates. Whether or not this is the smallest number of gates needed to represent a general U(4) group remains to be investigated.

Interestingly, by using this network of gates represented by the $U(4)$ box it is possible to get the most 
general 3 qubit network (U(8) group). The arrangement for this was given and discussed by Barenco et.al [12. The most general q qubit network $\left(U\left(2^{q}\right)\right.$ group $)$ can be achieved in a similar manner. However, the number of $\mathrm{U}(4)$ networks will grow exponentially with number of qubit lines q.

In all, cyclic quantum gate networks may be classified according to the structure of the unitary matrix representing the cyclic network's combined gate-operations for one iteration of the qubit(s). This is helpful when analyzing the evolution of simple cyclic networks because understanding the evolution of one group encompasses many different types of cyclic gate arrangements within that group. In the next section, the classification of these simple groups will be quite useful because it simplifies the eigenvalue and eigenstate analysis of cyclic networks to just those representing the most general group arrangements.

\section{EIGENVALUES AND EIGENSTATES}

One of the goals in studying cyclic quantum networks is to understand the evolution of the wavefunction for qubits cycling through the network. For example, the evolution of a cyclic network, such as in Figures 1 12 , take the form

$$
\Psi(n)=(U)^{n} \Psi(0)
$$

where $n$ is the number of cycles completed and $U$ represents the gate operations resulting from one cycle around the network.

In practice, it is convenient to express Equation 24 in the $U$ operator eigenbasis. The expansion of $\Psi$ in the $U$ eigenbasis will take the form

$$
\Psi(0)=\sum_{\mu=1}^{2^{q}} c_{\mu} \Psi_{\mu}
$$

where $\mathrm{q}$ is the number of qubit loops and $\Psi_{\mu}$ is the eigenstate corresponding to eigenvalue $e^{i \nu_{\mu}}$.

Use of Equation 25 shows that

$$
\Psi(n)=\sum_{\mu=1}^{2^{q}} c_{\mu} e^{i n \nu_{\mu}} \Psi_{\mu}
$$

\section{A. One Qubit Eigenvalues and Eigenstates}

¿From the classification of cyclic networks in the previous chapter it is understood that a general $\mathrm{U}(2)$ cyclic network with the matrix operator given by Equation 1 gives the U(2) network's evolution, in the eigenbasis as

$$
U^{n} \Psi=c_{1} e^{i n \nu_{1}} \Psi_{1}+c_{2} e^{-i n \nu_{1}} \Psi_{2}
$$

where $\nu_{2}=-\nu_{1}$. This also represents the evolution of $\mathrm{SU}(2)$ and $\mathrm{SO}(2)$ networks with appropriate restrictions of $\alpha, \beta, \delta$.

\section{B. Two Qubit Eigenvalues and Eigenstates}

As discussed in section II the most general two qubit network belongs to the $\mathrm{U}(4)$ group. It operates on all four basis states $|00\rangle,|01\rangle,|10\rangle,|11\rangle$, with a set of control gates and single qubit gates as shown in Equations 17 . 23. Since the main interest here is in $\mathrm{U}(3), \mathrm{SU}(3)$ and $\mathrm{SO}(3)$ networks, the discussion of the evolution of these networks will be limited to these groups.

\section{Eigenvalues for U(3) and Subgroups}

The evolution of a cyclic network belonging to U(3) or one of its subgroups is given by Equation 26. Replacing $U$ by $G$ from Equation 1 gives the following for $\Psi(n)=$ $G^{n} \Psi$ :

$$
G^{n} \Psi=c_{1} e^{i n \nu_{1}} \Psi_{1}+c_{2} e^{i n \nu_{2}} \Psi_{2}+c_{3} e^{i n \nu_{3}} \Psi_{3}+c_{4} e^{i n \nu_{4}} \Psi_{4}
$$

The 4 eigenvalues for $\mathrm{G}$ are $\lambda=1$ and 3 eigenvalues corresponding to the following characteristic equation of degree three

$$
\lambda^{3}+a_{1} \lambda^{2}+a_{2} \lambda+a_{3}=0
$$

The $a_{s}$ represent the principal minors [19] of the lower $3 \times 3$ matrix $M$ in $G$, Equation 1 .

The solution to this general cubic equation with real or complex coefficients may be found in references 22, 21, and is given to be

$$
\lambda_{k}=w^{\frac{1}{3}} e^{\frac{i k 2 \pi}{3}}-\frac{P}{3 w^{\frac{1}{3}} e^{\frac{i k 2 \pi}{3}}}-\frac{a_{1}}{3} \quad \text { For } \quad k=0,1,2
$$

where

$$
w=\frac{Q}{2}+\sqrt{\frac{Q^{2}}{4}+\frac{P^{3}}{27}}
$$

and

$$
Q=\frac{9 a_{1} a_{2}-27 a_{3}-2 a_{1}^{3}}{27} \quad ; \quad P=\frac{3 a_{2}-a_{1}^{2}}{3}
$$

The coefficients $a_{1}, a_{2}, a_{3}$ in the cubic equation are given by

$$
\begin{aligned}
& a_{1}=-\operatorname{Tr} M \\
& a_{2}=M_{11}+M_{22}+M_{33} \\
& a_{3}=-\operatorname{det} M
\end{aligned}
$$

where

$$
\begin{aligned}
& M_{11}=\operatorname{det}\left(\begin{array}{ll}
m_{22} & m_{23} \\
m_{32} & m_{33}
\end{array}\right) \\
& M_{22}=\operatorname{det}\left(\begin{array}{ll}
m_{11} & m_{13} \\
m_{31} & m_{33}
\end{array}\right) \\
& M_{33}=\operatorname{det}\left(\begin{array}{ll}
m_{11} & m_{12} \\
m_{21} & m_{22}
\end{array}\right)
\end{aligned}
$$




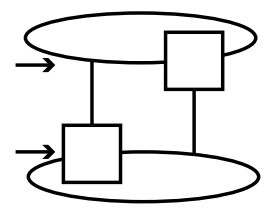

FIG. 3: An example of an SU(3) cyclic network with two alternating control gates of the form $G_{d n}(\alpha, \phi, \beta)$ and $G_{u p}(\alpha, \phi, \beta)$.

If we restrict the 2 qubit cyclic network from $\mathrm{U}(3)$ to an $\mathrm{SU}(3)$ by setting $\delta_{1}+\delta_{2}+\delta_{3}=0$ the coefficients $a_{1}, a_{2}, a_{3}$ can be immediately simplified to

$$
\begin{aligned}
& a_{1}=-\operatorname{Tr} M \\
& a_{2}=(\operatorname{Tr} M)^{*} \\
& a_{3}=-1
\end{aligned}
$$

The result for $a_{3}$ follows directly from the determinant 1 group property for the $\mathrm{SU}(3)$ group. However, the reduction $a_{2}=(\operatorname{Tr} M)^{*}$ is not as apparent. For this result it is helpful to realize that the cubic equation (Equation 29) may be rewritten as

$$
\left(\lambda-\lambda_{0}\right)\left(\lambda-\lambda_{1}\right)\left(\lambda-\lambda_{2}\right)=0
$$

giving the following relations for the coefficients $a_{s}$ :

$$
\begin{aligned}
& \lambda_{0}+\lambda_{1}+\lambda_{2}=-a_{1} \\
& \lambda_{0} \lambda_{1}+\lambda_{1} \lambda_{2}+\lambda_{0} \lambda_{2}=a_{2} \\
& \lambda_{0} \lambda_{1} \lambda_{2}=-a_{3}
\end{aligned}
$$

By noting that $\lambda_{0} \lambda_{1} \lambda_{2}=1$ for the $\mathrm{SU}(3)$ matrix and that the eigenvalues to the control gate networks are of the form $\lambda_{k}=e^{i \nu_{k}}$ as for all unitary matrices. It becomes evident that

$$
\lambda_{0} \lambda_{1}+\lambda_{1} \lambda_{2}+\lambda_{0} \lambda_{2}=\left(\lambda_{0}^{*}+\lambda_{1}^{*}+\lambda_{2}^{*}\right)
$$

showing that $a_{2}=(\operatorname{Tr} M)^{*}$.

A particular example of a cyclic network belonging to the $S U(3)$ group is the network with an alternating pair of gates $G_{u p}(\alpha, \phi, \beta) G_{d n}(\alpha, \phi, \beta)$ as in Figure 3. In this case, $\operatorname{Tr} M=A(\alpha, \phi)$ where

$$
A(\alpha, \phi)=e^{-2 i \alpha} \cos ^{2}(\phi)+2 e^{i \alpha} \cos (\phi) .
$$

¿From Equation 35 one has $a_{1}=-A(\alpha, \phi)$ and $a_{2}=$ $A^{*}(\alpha, \phi)$. Therefore, the eigenvalues are $\lambda=1$ and three other eigenvalues as the solutions to the cubic eigenvalue equation

$$
\lambda^{3}-A(\alpha, \phi) \lambda^{2}+A^{*}(\alpha, \phi) \lambda-1=0
$$

The solutions to the cubic equation follow from Equation 30 but can be simplified further by making use of the property $A\left(\alpha \pm \frac{2 \pi}{3}, \phi\right)=A(\alpha, \phi) e^{\frac{ \pm 2 \pi}{3}}$. This can be verified directly by substituting $\alpha \pm \frac{2 \pi}{3}$ into Equation 39. By

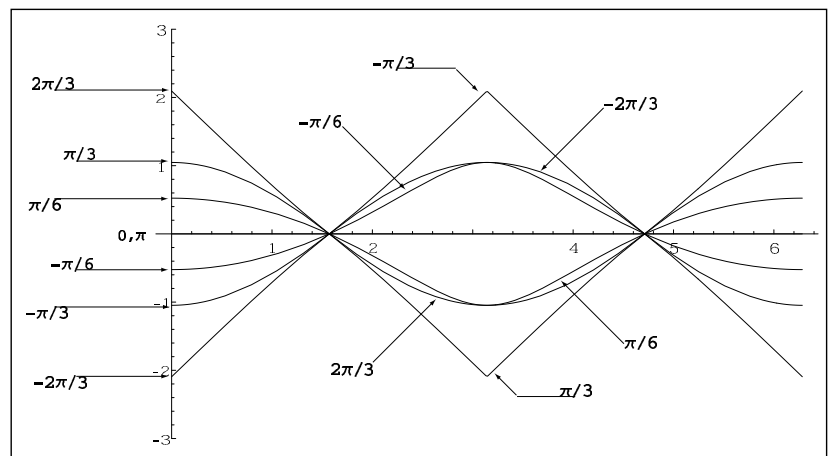

FIG. 4: A plot of eigenphase $\nu_{0}$ corresponding to the eigenvalue $\lambda_{0}=e^{i \nu_{0}}$ where the ordinate represents the value of $\nu_{0}$, the abscissa represents the value of $\phi$ and each of the family of curves in the plot are given for different $\alpha$.

making use of this property $w, Q, P$ from Equations 3132 have the following relations:

$$
\begin{aligned}
& w\left(\alpha \pm \frac{2 \pi}{3}, \phi\right)=w(\alpha, \phi) \\
& Q\left(\alpha \pm \frac{2 \pi}{3}, \phi\right)=Q(\alpha, \phi) \\
& P\left(\alpha \pm \frac{2 \pi}{3}, \phi\right)=P(\alpha, \phi) e^{\mp i 2 \pi} \frac{}{3}
\end{aligned}
$$

and the solution to the cubic equation (Equation 40) takes on the simple form:

$$
\lambda_{k}=\lambda_{0}\left(\alpha_{k}, \phi\right) e^{\frac{i k 2 \pi}{3}}
$$

where

$$
\lambda_{0}\left(\alpha_{k}, \phi\right)=w^{\frac{1}{3}}\left(\alpha_{k}, \phi\right)-\frac{p\left(\alpha_{k}, \phi\right)}{3 w^{\frac{1}{3}}\left(\alpha_{k}, \phi\right)}+\frac{A\left(\alpha_{k}, \phi\right)}{3}
$$

and

$$
\alpha_{k}=\alpha-\frac{2 k \pi}{3}
$$

As an example consider when $\alpha=\frac{\pi}{4}$, for this case the three eigenvalue solutions using Equation 42 are $\lambda_{0}=\lambda_{0}\left(\frac{\pi}{4}, \phi\right), \lambda_{1}=\lambda_{0}\left(\frac{\pi}{4}-\frac{2 \pi}{3}, \phi\right) e^{\frac{i 2 \pi}{3}}$ and $\lambda_{2}=$ $\lambda_{0}\left(\frac{\pi}{4}-\frac{4 \pi}{3}, \phi\right) e^{\frac{i 4 \pi}{3}}$.

Since the three eigenvalues may be extracted from the eigenvalue $\lambda_{0}$, it is sufficient to plot the eigenphase $\nu_{0}$ in order to analyze the properties of the three eigenvalues. A plot of eigenphase $\nu_{0}$ corresponding to the eigenvalue $\lambda_{0}=e^{i \nu_{0}}$ can be seen in Figure 1 where the ordinate represents the value of $\nu_{0}$, the abscissa represents the value of $\phi$ and each of the family of curves in the plot are given for different $\alpha$.

There are some important properties that can be noted about these eigenvalue solutions. The first property pertains to three special solutions that are constant for all values of $\phi$. These are the solutions for which the eigenphase $\nu=\alpha$ and can be attained by directly substituting 
$\lambda=e^{i \alpha}$ into Equation 40 and expanding out the $A(\alpha, \phi)$ as given by Equation 39. This gives the following equation

$$
\left(e^{3 i \alpha}-1\right)\left(\cos ^{2}(\phi)-2 \cos (\phi)-1\right)=0
$$

with three special solutions $\alpha=0, \frac{2 \pi}{3}, \frac{-2 \pi}{3}$ corresponding to $\lambda=1, e^{\frac{i 2 \pi}{3}}, e^{-\frac{i 2 \pi}{3}}$. (Note, that only one of these solutions appears in the plot and corresponds to $\lambda_{0}$ with $\nu_{0}=0$. The other two solutions are just a shift as described by Equation 42.) A second property is the symmetry $\lambda_{0}(-\alpha)=\lambda_{0}(\alpha)^{*}$ which can be seen in Figure as the eigenphase $\nu_{0}$ is symmetric about the $\nu_{0}=0$ axis. This can also be verified directly in Equation 43 by noticing that $p, w$ have this symmetry due to the fact that $A(-\alpha, \phi)=A^{*}(\alpha, \phi)$. Similarly, a third property associated with the eigenvalues of this particular $\mathrm{SU}(3)$ network is the translational symmetry $\lambda(\alpha+\pi, \phi)=\lambda(\alpha, \phi+\pi)$ which can also be directly associated to $A(\alpha, \phi)$ having the same translational symmetry. As a final comment on the $\nu_{0}$ plot, the nodes appearing at the values $\phi=\frac{\pi}{2}$ and $\phi=\frac{3 \pi}{2}$ occur because $A\left(\alpha, \frac{\pi}{2}\right)=0$ and $A\left(\alpha, \frac{3 \pi}{2}\right)=0$ for all values of $\alpha$. A simple examination of Equation 40 shows that for these two values of $\phi$, $\lambda_{0}=1$ for all $\alpha$.

An interesting variation on this example is when $-\alpha$ appears in one of the two alternating gates such as in the case $G_{u p}(-\alpha, \phi, \beta) G_{d n}(\alpha, \phi, \beta)$. The trace $\mathrm{M}$ of this network now becomes

$$
\operatorname{Tr} M=2 \cos (\alpha) \cos (\phi)+\cos ^{2}(\phi)
$$

and the coefficients to the cubic equation are now real and take on the relationship $a_{2}=-a_{1}$. Cases like this where the trace is real simplify the solution to the form

$$
\lambda_{0}=1, \quad \lambda_{1,2}=\frac{\operatorname{Tr} M-1}{2} \pm \frac{i \sqrt{(3-\operatorname{Tr} M)(\operatorname{Tr} M+1)}}{2}
$$

where

$$
\cos \nu=\frac{\operatorname{Tr} M-1}{2}, \quad \sin \nu=\frac{\sqrt{(3-\operatorname{Tr} M)(\operatorname{Tr} M+1)}}{2}
$$

Other cases where the network's trace is real belong to cyclic networks with just a single Control-SU(2) or Control-SO(2) gate as in Figure 2(b). This of course is trivial, and for that matter the eigenvalues are the same as that in the single qubit case. Of more interest is the general $S O(3)$ group. In this case, the three gates are $G_{u p}\left(\phi_{1}\right) G_{d n}\left(\phi_{2}\right) G_{u p}\left(\phi_{3}\right)$ and the trace of the network has the simple form

$$
\operatorname{Tr} M=\cos \left(\phi_{2}\right)+\cos \left(\phi_{1}+\phi_{3}\right)\left(1+\cos \left(\phi_{2}\right)\right)
$$

In the next section a cyclic control gate network belonging to the $S O(3)$ group will be used as an example to understand the perturbative effects of an acyclic line acting on the cyclic network.

\section{Eigenstates of $U(3)$ and Subgroups}

It is fairly simple to write down the 4 unnormalized qubit eigenstates for control gate cyclic networks. The following unnormalized eigenstates can be applied to any network with matrix-gate structure G (Note: G matrix structure includes $\mathrm{U}(3)$ and subgroups)

$$
\begin{gathered}
\Psi_{k}=\left(\begin{array}{cc}
0 & |00\rangle \\
-m_{13}\left(m_{22}-\lambda_{k}\right)+m_{12} m_{23} & |01\rangle \\
-m_{23}\left(m_{11}-\lambda_{k}\right)+m_{21} m_{13} & |10\rangle \\
\left(m_{22}-\lambda_{k}\right)\left(m_{11}-\lambda_{k}\right)-m_{21} m_{12}
\end{array}\right) \\
|11\rangle \\
\Psi_{3}=\left(\begin{array}{l}
1 \\
0 \\
0 \\
0
\end{array}\right) \begin{array}{l}
|00\rangle \\
|01\rangle \\
|10\rangle \\
|11\rangle
\end{array}
\end{gathered}
$$

$\Psi_{k}$ corresponds to eigenstates $k=0,1,2$ and $\lambda_{k}$ are the eigenvalues associated with the respective eigenstates.

\section{PERTURBATION OF A CYCLIC NETWORK}

Some areas of investigation that may lead to potential applications for cyclic quantum networks are quantum memories, quantum sensors and the ability to make existing acyclic quantum gate arrays more compact. As a first step in understanding the feasibility of these potential applications, it is essential to be able to interact with the cyclic network via qubit(s) on an acyclic line. A very straightforward method of interacting with a cyclic network is to connect the cyclic network to an acyclic qubit via a control gate as seen in Figure 5(a,b).

In the rest of this section an analysis of a general two qubit cyclic network perturbed by an acyclic qubit will be examined. Following this general description, a specific example will be presented. (Note: The analysis of a perturbed single qubit cyclic network can be readily achieved by following the outlined method for the perturbed two qubit case.)

\section{A. Perturbation of a Two Qubit Cyclic Network}

In examining the two qubit cyclic network in Figure $5(\mathrm{a}, \mathrm{b})$, the acyclic line is connected to the bottom cyclic loop via a Control-Not gate. Box "U" in the cyclic network denotes any arrangement of quantum gates, to include $\mathrm{U}(4)$ and its subgroups. Prior to an interaction with the acyclic qubit, the cyclic network evolves as previously described in section III. However, after an interaction the cyclic network evolves in a perturbed/entangled state with the acyclic qubit. (Note: the acyclic qubit, and the bottom, cyclic qubit must be coincident at the Control-Not gate at the time of interaction). The acyclic qubit, which remains in an entangled state with the cyclic 
(a)

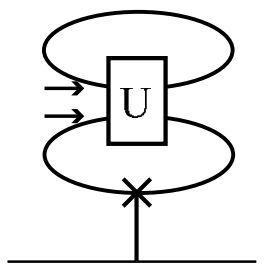

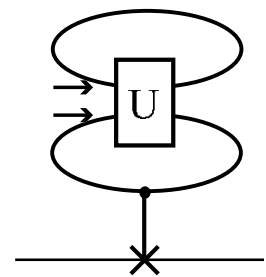

(b)
FIG. 5: Two qubit cyclic network perturbed by an acyclic qubit via a Control-Not gate. Note, the box "U" denotes any set of gates acting in the 4 dimensional Hilbert Space.

network, proceeds past the interaction gate as the cyclic qubits continue to move around the network.

To examine this more closely, the following requirements are to be considered. First, the state of the qubits in the cyclic network is initially set to an arbitrary superposition

$$
\Psi=c_{1}|00\rangle+c_{2}|01\rangle+c_{3}|10\rangle+c_{4}|11\rangle
$$

Second, the cyclic network is allowed to evolve an arbitrary number of $n$ iterations before it interacts with the acyclic qubit. Third, an acyclic qubit in the arbitrary state

$$
\Phi=\alpha|0\rangle+\beta|1\rangle
$$

interacts with the cyclic network via a Control-Not gate, and then continues its course on the acyclic line as the qubits in the cyclic network continues to evolve for $n^{\prime}$ more iterations. In considering a case like this the resulting state is

$$
\left\{P_{0} \otimes U^{\left(n^{\prime}+n\right)}+P_{1} \otimes U^{n^{\prime}} \sigma_{x, b} U^{n}\right\}|\Phi \otimes \Psi\rangle
$$

for Figure $5(\mathrm{a})$. To write this equation the relation $(1 \otimes U) P_{1} \otimes \sigma_{x, b}=P_{1} \otimes U \sigma_{x, b}$ has been used. The $P_{0}=|0\rangle\langle 0|$ and $P_{1}=|1\rangle\langle 1|$ are projection operators on the state of the perturbing qubit, $\sigma_{x}$ represents a Pauli Matrix and the operator $\mathrm{U}$ is an arbitrary matrix which represents the gate operation of the cyclic network upon one iteration. Note, that the subscript b associated with the operators represent the bottom qubit of the cyclic network.

If the Control-Not gate connected to the acyclic line is flipped around so as to have the target bit on the acyclic line and the control on the cyclic line as in Figure 5(b), the resulting state evolution then becomes

$$
\left\{1 \otimes U^{n^{\prime}} P_{0, b} U^{n}+\sigma_{x} \otimes U^{n^{\prime}} P_{1, b} U^{n}\right\}|\Phi \otimes \Psi\rangle .
$$

To write this equation the relation $U \sigma_{x} \otimes P_{1, b}=\sigma_{x} \otimes$ $U P_{1, b}$ has been used. One aspect to note about this analysis is that the Control-Not gate may easily be replaced by a more general Control-U(2) gate by simply changing the $\sigma_{x}$ for the more general $\mathrm{U}(2)$ operator. by

The matrix elements of $U^{n}$ in the binary basis are given

$$
\left\langle j^{\prime}\left|U^{n}\right| j\right\rangle=\sum_{\mu}\left|j^{\prime}\right\rangle\left\langle j^{\prime}|| \Psi_{\mu}\right\rangle\left\langle\Psi_{\mu} \mid j\right\rangle\langle j| e^{i n \nu_{\mu}}
$$

where $U^{n} \Psi_{\mu}=e^{i n \nu_{\mu}} \Psi_{\mu}$ has been used. This is useful in further examining Equations 53 and 54 .

\section{B. Example of a Perturbed Two Qubit Cyclic Network}

For this example we restrict ourselves to an $\mathrm{SO}(3)$ cyclic network because it shows the essential properties for a network undergoing an acyclic qubit perturbation. Using Equation 53 an $\mathrm{SO}(3)$ cyclic network perturbed by an acyclic qubit, such as in Figure 5(a), may be examined. In this case, $U=G$ and the operation of the cyclic network on the cyclic qubits upon one iteration will be described by

$$
G=G_{u p}(\phi) G_{d n}(\phi)
$$

where $G_{u p}(\phi)$ and $G_{d n}(\phi)$ are defined in Equations 2 and 3 with $\alpha, \beta, \delta=0$.

For $G^{n}$ iterations the matrix elements are given as a function of $n$ iterations with the aid of Equation 55 . One sees from Equation 1 that it is sufficient to consider the lower $3 \times 3$ matrix M. The matrix elements $m_{j, j^{\prime}}=m_{j, j^{\prime}}(n)$ have the form

$$
A(\phi)+B(\phi) \operatorname{cosn} \nu_{1}+C(\phi) \operatorname{sinn} \nu_{1}
$$

where the coefficients $A(\phi), B(\phi), C(\phi)$ for each of the $m_{j, j^{\prime}}$ are given in Table [. (Table I] uses the following abbreviations $c=\cos \phi$ and $s=\sin \phi$.) Note, that only the eigenphase $\nu_{1}$ appears in Equation 57, this is due to the fact that $\nu_{0}=\nu_{3}=1$ and $\nu_{2}=-\nu_{1}$ as for all $\mathrm{SO}(3)$ matrices.

With the aid of these matrix elements it is now possible to solve for the evolution of the network after its acyclic qubit interaction. In order to simplify this example the cyclic network will initially be placed in one of its eigenstates instead of an arbitrary superposition as in Equation 51. Using Equation 50 in section III, the eigenstates of $\mathrm{G}$ are

$$
\begin{gathered}
\Psi_{k}=N_{k}\left(\begin{array}{c}
0 \\
-\sin (\phi)\left(1-\lambda_{k} \cos (\phi)\right) \\
-\sin (\phi)\left(\cos (\phi)-\lambda_{k}\right) \\
\left(\cos (\phi)-\lambda_{k}\right)^{2}
\end{array}\right) \begin{array}{l}
|00\rangle \\
|01\rangle \\
|10\rangle \\
|11\rangle
\end{array} \quad k=0,1,2 \\
\Psi_{3}=\left(\begin{array}{l}
1 \\
0 \\
0 \\
0
\end{array}\right) \begin{array}{l}
|00\rangle \\
|01\rangle \\
|10\rangle \\
|11\rangle
\end{array}
\end{gathered}
$$




\begin{tabular}{c|c|c|c}
\hline$m_{j, j^{\prime}}$ & $A(\phi)$ & $B(\phi)$ & $C(\phi)$ \\
\hline \hline$m_{1,1}$ & $\frac{(c+1)}{(c+3)}$ & $\frac{2}{(c+3)}$ & 0 \\
\hline$m_{1,2}$ & $\frac{-(c+1)}{(c+3)}$ & $\frac{4 c-2 c^{2} \cos \nu-2 \cos \nu_{1}}{s^{2}(c+1)(c+3)}$ & $\frac{-2 \sin \nu_{1}}{(c+1)(c+3)}$ \\
\hline$m_{1,3}$ & $\frac{-s}{(c+3)}$ & $\frac{2 c^{3} \cos \nu_{1}-6 c^{2}+6 c \cos \nu_{1}-2 \cos 2 \nu_{1}}{s^{3}(c+1)(c+3)}$ & $\frac{-2 c^{3} \sin \nu_{1}+6 c \sin \nu_{1}-2 \sin 2 \nu_{1}}{s^{3}(c+1)(c+3)}$ \\
\hline$m_{2,1}$ & $\frac{-(c+1)}{(c+3)}$ & $\frac{4 c-2 c^{2} \cos \nu_{1}-2 \cos \nu_{1}}{s^{2}(c+1)(c+3)}$ & $\frac{2 \sin \nu_{1}}{(c+1)(c+3)}$ \\
\hline$m_{2,2}$ & $\frac{(c+1)}{(c+3)}$ & $\frac{2}{(c+3)}$ & 0 \\
\hline$m_{2,3}$ & $\frac{s}{(c+3)}$ & $\frac{2\left(-c^{3}+3 c^{2} \cos \nu_{1}-c\left(\cos 2 \nu_{1}+2\right)+\cos \nu_{1}\right)}{s^{3}(c+1)(c+3)}$ & $\frac{2\left(c^{2} \sin \nu_{1}-c \sin 2 \nu_{1}+\sin \nu_{1}\right)}{s^{3}(c+1)(c+3)}$ \\
\hline$m_{3,1}$ & $\frac{-s}{(c+3)}$ & $\frac{2 c^{3} \cos \nu_{1}-6 c^{2}+6 c \cos \nu_{1}-2 \cos 2 \nu_{1}}{s^{3}(c+1)(c+3)}$ & $\frac{2 c^{3} \sin \nu_{1}-6 c \sin \nu_{1}+2 \sin 2 \nu_{1}}{s^{3}(c+1)(c+3)}$ \\
\hline$m_{3,2}$ & $\frac{s}{(c+3)}$ & $\frac{2\left(-c^{3}+3 c^{2} \cos \nu_{1}-c\left(\cos 2 \nu_{1}+2\right)+\cos \nu_{1}\right)}{s^{3}(c+1)(c+3)}$ & $\frac{2\left(-c^{2} \sin \nu_{1}+c \sin 2 \nu_{1}-\sin \nu_{1}\right)}{s^{3}(c+1)(c+3)}$ \\
\hline$m_{3,3}$ & $\frac{(1-c)}{(c+3)}$ & $\frac{2(c+1)}{(c+3)}$ & 0 \\
\hline
\end{tabular}

TABLE I: Coefficients $A(\phi), B(\phi), C(\phi)$ for matrix elements $m_{j, j^{\prime}}$ in $G^{n}$. Note, $c=\cos \phi$ and $s=\sin \phi$.

with the following normalization factors

$$
\begin{aligned}
N_{0} & =\frac{1}{(1-\cos \phi) \sqrt{(1-\cos \phi)(\cos \phi+3)}} \\
N_{1,2} & =\frac{1}{\sin ^{2} \phi \sqrt{(\cos \phi+1)(\cos \phi+3)}}
\end{aligned}
$$

Since this cyclic network has been restricted to the $\mathrm{SO}(3)$ group its eigenvalues can be found from Equation 47 with the trace being set to

$$
\operatorname{Tr} M=\cos ^{2}(\phi)+2 \cos (\phi)
$$

In using Equation 53 to give the evolution of the cyclic network after the perturbation, we set the initial iterations of the cyclic network to $n=0$. This is due to the fact that the initial state of the cyclic network is in an eigenstate of $G$ and gives an inconsequential global phase for the $G^{n}$ iterations prior to the perturbation. Therefore, the evolution of the cyclic network after the perturbation is given by

$$
\alpha|0\rangle \otimes e^{i n^{\prime} \nu_{k}}\left|\Psi_{k}\right\rangle+\beta|1\rangle \otimes G^{n^{\prime}} \sigma_{x, b}\left|\Psi_{k}\right\rangle
$$

where the final state is found to be entangled into a superposition of an unperturbed and perturbed cyclic network state.

The unperturbed part of the final state is $\alpha|0\rangle \otimes$ $e^{i n^{\prime} \nu_{k}}\left|\Psi_{k}\right\rangle$ which evolves according to the phase $e^{i n^{\prime} \nu_{k}}$. In contrast, the perturbed part of the entangled state is $\beta|1\rangle \otimes G^{n^{\prime}} \sigma_{x, b}\left|\Psi_{k}\right\rangle$ and can be expanded out in the binary basis as

$$
\beta|1\rangle \otimes N_{k}\left(\begin{array}{cc}
-s\left(1-c \lambda_{k}\right) & |00\rangle \\
m_{1,2}\left(n^{\prime}\right)\left(c-\lambda_{k}\right)^{2}-s m_{1,3}\left(n^{\prime}\right)\left(c-\lambda_{k}\right) & |01\rangle \\
m_{2,2}\left(n^{\prime}\right)\left(c-\lambda_{k}\right)^{2}-s m_{2,3}\left(n^{\prime}\right)\left(c-\lambda_{k}\right) & |10\rangle \\
m_{3,2}\left(n^{\prime}\right)\left(c-\lambda_{k}\right)^{2}-s m_{3,3}\left(n^{\prime}\right)\left(c-\lambda_{k}\right) & |11\rangle \\
k=0,1,2 &
\end{array}\right.
$$

and

$$
\beta|1\rangle \otimes N_{k}\left(\begin{array}{c}
0 \\
m_{1,1}\left(n^{\prime}\right) \\
m_{2,1}\left(n^{\prime}\right) \\
m_{3,1}\left(n^{\prime}\right)
\end{array}\right) \begin{aligned}
& |00\rangle \\
& |01\rangle \\
& |10\rangle \\
& |11\rangle
\end{aligned} \quad k=3
$$

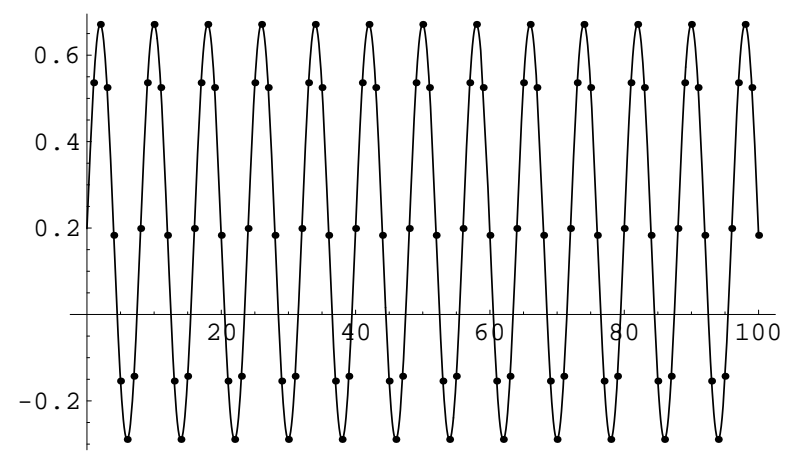

FIG. 6: The coefficient for the $|110\rangle$ basis state as a function of $n^{\prime}$ iterations where $\nu_{1}=\frac{\pi}{4}$.

¿From this it can be seen that the coefficient for $|100\rangle$ (leftmost digit belongs to the acyclic qubit) does not evolve in time with iteration number $n^{\prime}$. However, the coefficients for the other three basis states $|101\rangle,|110\rangle$ and $|111\rangle$ do evolve as a function of iteration number $n^{\prime}$. Furthermore, each of the coefficients for the basis states have the form

$$
A(\phi)+B(\phi) \operatorname{cosn}^{\prime} \nu_{1}+C(\phi) \operatorname{sinn}^{\prime} \nu_{1}
$$

due to the fact that all of the $m_{j, j^{\prime}}$ in Table 1 carry this form. (Note: $A(\phi), B(\phi)$ and $C(\phi)$ for the basis states may be complex for initial cyclic network eigenstates corresponding to $\mathrm{k}=1,2$ )

As an example of the perturbed evolution consider the case where the initial eigenstate is set to $k=0$ and the cyclic gate parameter $\phi$ is chosen so that $\nu_{1}=\frac{\pi}{4}$. For this case, the coefficient of the basis state $|110\rangle$ in Equation 62 will evolve as shown in Figure 6 . The coefficients for the other two basis states $|101\rangle$ and $|111\rangle$ are similar in nature. The ordinate represents the probability amplitude and the abscissa corresponds to the time interval $n^{\prime}$. The evolution of the coefficient can be seen on the plot as points on the continuous curve. The continuous curve is just a background which represents Equation 64 


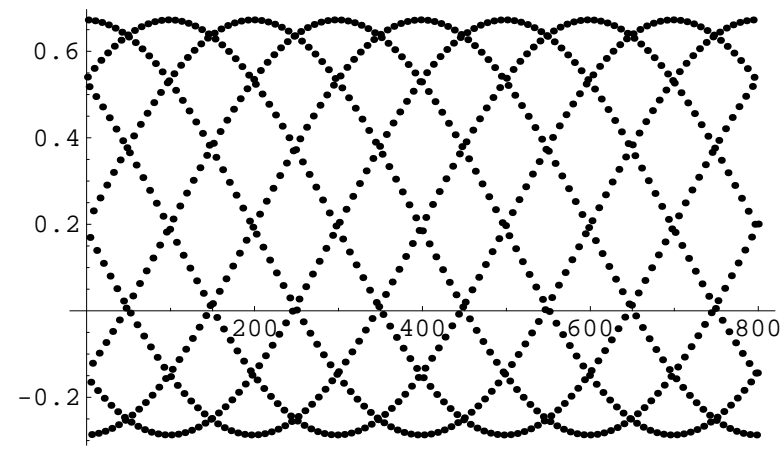

FIG. 7: The coefficient for the $|110\rangle$ basis state as a function of $n^{\prime}$ iterations where $\nu_{1}=\frac{(\pi+.01 \pi)}{4}$.

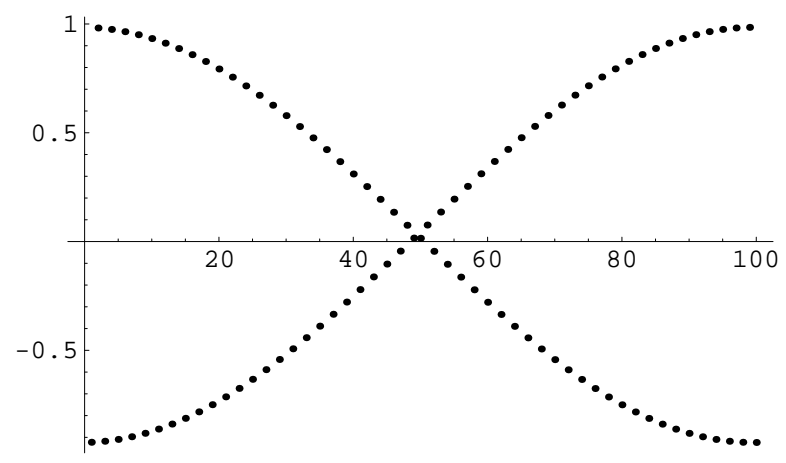

FIG. 8: The coefficient for the $|110\rangle$ basis state as a function of $n^{\prime}$ iterations where $\nu_{1}=.99 \pi$.

with $n^{\prime}$ continuous, and it does not represent the evolution of the cyclic network's qubits because the state of the qubits evolve in discrete time steps. It is only shown here to illustrate how the perturbed cyclic network's evolution samples the continuous curve and to help explain the next figure where a small deviation is made to $\nu_{1}=\frac{\pi}{4}$.

One interesting feature for the evolution corresponding to $\nu_{1}=\frac{\pi}{4}$ is when a small deviation is made, such as $\nu_{1}=\frac{(\pi+.01 \pi)}{4}$. For this case, Figure 7 illustrates how each of the points in one period of the continuous curve is modulated by a period of 800 iterations. In other words, if we choose one of the points in Figure 6 and modulate it by the $\frac{.01 \pi}{4}$ deviation the point will follow one of the iterated curves in Figure 7 for 800 iterations before returning to its original value. This may be understood by rewriting Equation 64, for $n^{\prime}$ integer, as

$$
\left.A(\phi)+B(\phi) \operatorname{cosn}^{\prime}\left(\frac{\pi}{4}+\frac{.01 \pi}{4}\right)+C(\phi) \operatorname{sinn}^{\prime}\left(\frac{\pi}{4}+\frac{.01 \pi}{4}\right)\right)
$$

where the factor $\frac{.01 \pi}{4}$ modulates the equation and does not complete a period until $n^{\prime}=800$.

Another interesting example of this type of modulation is when $\nu_{1}=.99 \pi$ as can be seen in Figure 8 for basis state $|110\rangle$. In this case the points are modulated in a similar manner but the iteration period is $n^{\prime}=200$.
Note, the period may easily be extended by a factor of 10 by simply choosing $\nu_{1}=.999 \pi$. The fact that the periodicity of these basis states may be increased in this way may have potential applications in making cyclic network sensors.

As a final comment for this particular example of a perturbed cyclic network, it's worth mentioning that for initial cyclic network states $\Psi_{0}, \Psi_{1}$ and $\Psi_{2}$ the perturbed part of the entangled wavefunction goes into a superposition of its four eigenstates. However, for an initial state starting in $\Psi_{3}$ the perturbed part goes into a superposition of three eigenstates excluding the original $\Psi_{3}$. This property will also be discussed in the next section as a possible application for a cyclic network memory, or sensor.

\section{DISCUSSION}

The classification and evolution of one and two qubit cyclic quantum networks for unperturbed and perturbed systems have been addressed. Up to now, mainstream quantum information research has focused on the goal of building a large quantum computer for factoring or searching. One possible spin off from this type of research might be quantum sensors and quantum memories that are only a few qubits in length. For this type of quantum information application, one or two qubit cyclic quantum networks may be well suited. This is not only due to the small number of qubits but also to the simplicity of the repeating gate operations. The fact that cyclic repetitions may be simpler to implement experimentally than complicated, varying gate arrangements, may be an advantage to current experimental efforts. Other interesting possibilities lie in the exploration of quantum algorithms and quantum information processing with cyclic quantum networks.

In the next sections a discussion about these possible research directions and applications of cyclic quantum networks will follow.

\section{A. Quantum Algorithms and Quantum Information Processing with Cyclic Quantum Networks}

As mentioned earlier, the quantum phase estimation algorithm can easily be expressed more compactly with cyclic quantum networks. For instance, Figure 9 shows the phase estimation algorithm as typically depicted with an acyclic array [4]. It also shows the equivalent cyclic network with the acyclic qubits acting in a conditional manner on the cyclic network's unitary operation.

Although expressing the quantum phase estimation algorithm in this way is quite trivial and suggests no new algorithms, it does serve as an example of a compact, cyclic network with an acyclic qubit line (similar to the ones investigated in the last section). Another example of an algorithm that can be compactly expressed in 
$|\mathrm{u}\rangle$
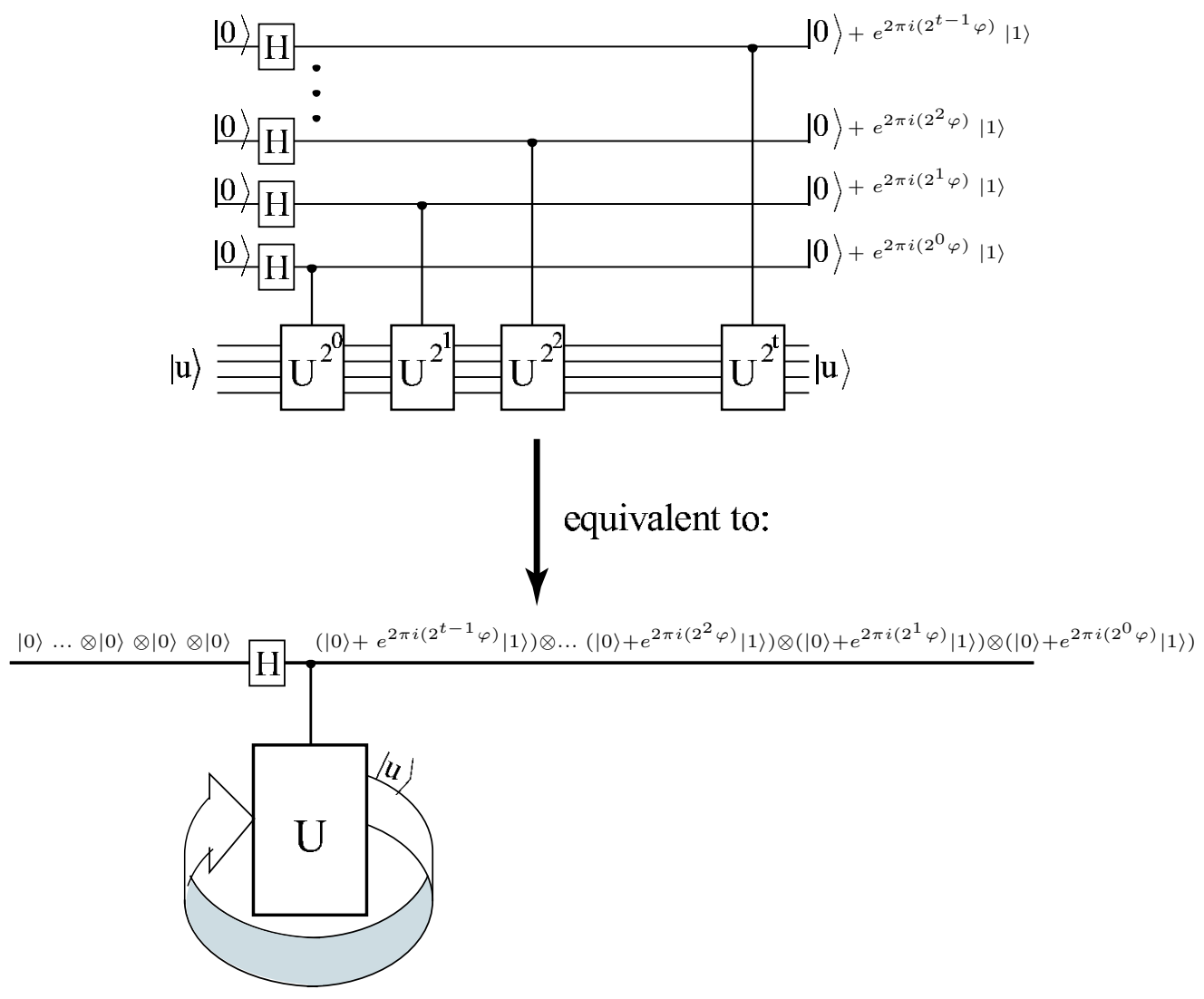

FIG. 9: The acyclic network at the top is the phase estimation network which is equivalent to the cyclic network shown below it. The operator $\mathrm{U}$ has eigenstate $|u\rangle$ with corresponding eigenvalue $e^{2 \pi i \phi}$. Note, the cyclic network must evolve for $U^{2^{t}}$ times for the $t$-th acyclic qubit crossing the acyclic control line. The single qubit operator $\mathrm{H}$ puts $|0\rangle$ into an equal superposition $\left.\frac{1}{\sqrt{2}}(|| 0\rangle+|1\rangle\right)$ where the $\frac{1}{\sqrt{2}}$ terms have been left off the diagram.

terms of cyclic networks is Grover's Search Algorithm [2. This is due to the repeating unitary operation sometimes called Grover's iterate which can also be modelled by a cyclic network. This common pattern of iterative operations in quantum algorithms is reminiscent of the observation that quantum algorithms resemble a multiparticle interferometer [4]. This observation (quantum algorithms resembling multiparticle interferometers) supports the finding that many quantum algorithms including Shor's algorithm may be viewed as a phase estimation process. It is possible that continued investigations into the iterative nature of these algorithms viewed in terms of cyclic networks may bring new ideas into quantum algorithm design.

Aside from expressing quantum algorithms with cyclic networks, one possible scope of applications for simple cyclic networks might involve methods of connecting them to existing acyclic arrays. These type of connections could potentially act as a type of subroutine for the acyclic arrays, in that the known perturbed evolution for the cyclic networks might serve as a type of module algorithm within the whole array.

Other applications may emerge from finding ways of connecting these simple cyclic networks together in

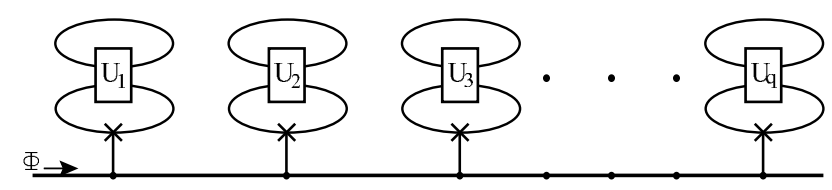

FIG. 10: A chain network comprised of two qubit cyclic networks linked by one acyclic line.

chains so as to understand the evolution of the overall composite network from the known state-evolution of the simpler networks. In other words, the evolution of qubits belonging to a group $\mathrm{U}\left(2^{q}\right)$ may be understood by simply writing down the wavefunction for the system which is comprised of the wavefunctions for the simpler cyclic networks. This type of analysis could potentially bring on new ways of understanding quantum information processing for large $2^{q}$ dimensional Hilbert Spaces.

One example of a composite cyclic network belonging to the $\mathrm{U}\left(2^{q}\right)$ group can be seen in the chain network of Figure 10. This network obviously gives a highly entangled number of qubits. The total network is arranged so that each cyclic network's qubits move around the loops in one time step and cross the control gates in unison. 
Also, only one time step (iteration of qubit) occurs for the total network when the acyclic qubit moves from interacting with one cyclic network to interacting with the neighboring network. The total network's qubit evolution after the acyclic qubit $\Phi$ passes all the cyclic networks in q steps is given by

$$
\begin{gathered}
\left\{P_{0} \otimes\left[U_{q}^{\left(n^{\prime}+q\right)} \otimes \ldots \otimes U_{3}^{\left(n^{\prime}+q\right)} \otimes U_{2}^{\left(n^{\prime}+q\right)} \otimes U_{1}^{\left(n^{\prime}+q\right)}\right]\right. \\
+P_{1} \otimes U_{q}^{n^{\prime}} \otimes \ldots \otimes U_{3}^{n^{\prime}} \otimes U_{2}^{n^{\prime}} \otimes U_{1}^{n^{\prime}}\left[\sigma_{x, b}^{q} U_{q}^{q} \otimes \ldots \otimes U_{3}^{(q-2)} \sigma_{x, b}^{3} U_{3}^{2}\right. \\
\left.\left.\otimes U_{2}^{(q-1)} \sigma_{x, b}^{2} U_{2}^{1} \otimes U_{1}^{q} \sigma_{x, b}^{1}\right]\right\} \\
\quad\left|\Phi \otimes \psi_{q} \otimes \ldots \otimes \psi_{3} \otimes \psi_{2} \otimes \psi_{1}\right\rangle \quad(66)
\end{gathered}
$$

where the superscripts in $\sigma_{x, b}$ denote the cyclic network being operated on, and $n^{\prime}$ corresponds to the number of iterations after the perturbation of the cyclic networks. Note, the $P_{0}$ and $P_{1}$ operate on the acyclic qubit.

As a final remark on compact cyclic networks, it should be mentioned that chains of cyclic networks may be useful in the field of neural networks. For instance, by connecting just a few input and output lines to a cyclic network (or a chained set of cyclic networks), one can begin to ask questions on how this network might be trained in the same sense that the highly cyclical neural networks are trained 22, 23]. One simple method might rely on making measurements on the output qubits followed by a feedback of adjustments to the control gate parameters. The network can then be re-tested to see how the changes affect a new set of inputs. Simple procedures of this type using cyclic networks of quantum gates may be worth exploring in that it may benefit research in the field of neural networks.

\section{B. Quantum Memories}

One of the original motivations for studying cyclic quantum networks was the fact that quantum algorithms typically mention quantum memories, but give only one way of achieving this type of memory. This method implicitly relies on a system where no quantum operations act on a set of qubits (or a quantum register) until it is needed. Therefore, the quantum information in a set of qubits can be retained by sheltering the qubits from any interactions that may cause the information to be changed or lost.

An alternative to this type of quantum memory will be suggested in this section, but before doing this, it is helpful to re-cast the typical quantum memory into cyclic quantum circuit language. This is not difficult to do because it only involves setting the qubits (or register) into a cyclic network where the only operation being applied is the identity operation. In other words, the cyclic operators in Figures 2 are replaced by an identity operator whereby the qubits cycle about in an unaffected fashion.

In section III a discussion of all the one and two qubit groups of operators and their corresponding eigenstates and eigenvalues was given. These cyclic networks can potentially be used as quantum memories due to the fact that a set of qubits in an eigenstate of the cyclic network is unaltered (except for an inconsequential global phase) upon passing through the gates. Therefore, quantum information in the form of an eigenstate may be stored in a cyclic network.

However, if an arbitrary state expanded over the eigenstates of the cyclic network undergoes iterations, then the phases do become relevant. In this case, the differences of phases between the eigenstates act as interference terms in the binary basis altering the initial state of the memory. Nevertheless, the initial state may be recovered (in a reversible sense) due to the fact that the number of iterations $n$ that the cyclic network has evolved since the memory was saved is known and that the unitary operation $U$ for which the cyclic network operates on the qubits for one iteration is also known.

Consider the scheme described in Figure 11(a). In this description of a cyclic quantum memory, two qubits in an unknown quantum state $|\Psi(0)\rangle$ are saved in the cyclic quantum memory by swapping with the two qubits in the cyclic network $\mathrm{G}$ for which the parameters $m_{j, j^{\prime}}$ in $\mathrm{G}$ are known. The unknown quantum state is allowed to evolve $n$ iterations corresponding to the time the unknown quantum state is saved in the cyclic memory. After $\mathrm{n}$ iterations the unknown quantum state may be retrieved as in Figure 11.(b) by bringing two new qubits in the state $\left|\Phi^{\prime}\right\rangle$ and swapping with the evolved, unknown quantum state $|\Psi(n)\rangle$ in the cyclic network. The state $|\Psi(n)\rangle$ may be reversibly set back to its initial state $|\Psi(0)\rangle$ by applying the operation $\left(G^{\dagger}\right)^{n}$. Interestingly, this does not require n iterations of $G^{\dagger}$ due to the fact that Equation 55 may be used to generate the matrix elements to $G^{\prime}=\left(G^{\dagger}\right)^{n}$. Therefore, by applying the single operation $G^{\prime}$, the initial unknown quantum state may be retrieved.

In summary, this scheme allows cyclic networks to implement a novel quantum memory, but whether or not this type of quantum memory is of any use in quantum algorithm design or implementation remains an open question.

\section{Quantum Sensors}

In the context of cyclic quantum gate networks perturbed by an acyclic qubit, a quantum sensor may be defined. This is not difficult to achieve since a quantum sensor is nothing more than a recorder of an interaction. Furthermore, since the last section discussed methods of implementing a quantum memory via cyclic quantum gate networks, the quantum sensor can be thought of as a type of quantum memory recording a qubit interaction with the cyclic network.

One specific example of a quantum sensor that may be analyzed is the example of a perturbed two qubit cyclic network in section IV-B. If the goal of the quantum sensor is to be able to detect the passage of a qubit in state 


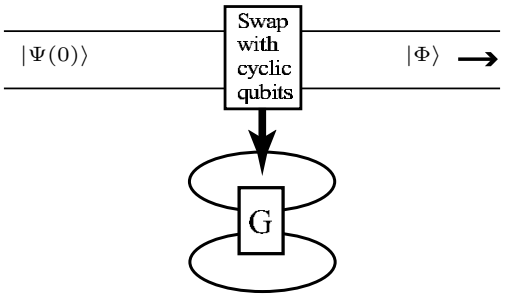

(a)

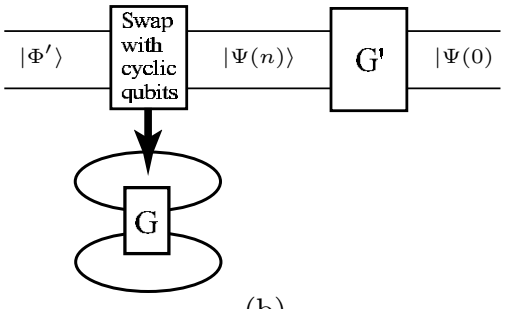

(b)

FIG. 11: (a)- Two qubits in an unknown quantum state $|\Psi(0)\rangle$ are placed into the cyclic quantum memory by swapping with two qubits in an arbitrary state $|\Phi\rangle$ initially in the cyclic network G. (b)- After saving the unknown quantum state for $n$ iterations, two new qubits in arbitrary state $\left|\Phi^{\prime}\right\rangle$ are swapped with the evolved, quantum state $|\Psi(n)\rangle$. The state is returned to its initial value by applying the inverse operation $G^{\prime}$.

$|1\rangle$ on the acyclic line, then a measurement of the cyclic network's qubit states can give this information if the cyclic network's evolution is perturbed from its unperturbed evolution.

One simple case for this is the cyclic network with initial state chosen to be $\Psi_{3}$. If an acyclic qubit state $|1\rangle$ interacts with the cyclic network via a Control-Not gate, the cyclic network goes into a perturbed superposition state of $\Psi_{0}, \Psi_{1}, \Psi_{2}$ and excludes the initial state $\Psi_{3}$ as mentioned in Section IV-B. A simple measurement to detect $\Psi_{3}$ can determine whether or not an acyclic qubit state $|1\rangle$ has interacted with the cyclic network.

This example on its own is not very impressive since a simple Control-Not gate can give this same type of information without having to make use of a cyclic network. However, a quantum sensor as a perturbed cyclic quantum network gives a method of recording an interaction where the recording qubits undergo repeated unitary operations. This type of sensor may be useful in experimen- tal situations where it is simpler to have repeated gate operations.

Another reason that cyclic quantum sensors may be of use, is in the possibility that quantum algorithms may be applied to the sensing process and thereby increasing capabilities beyond that of "classical" devices. This however remains to be investigated.

\section{CONCLUSION}

The structure of one and two qubit cyclic quantum gate networks have been classified. The unperturbed evolution for these networks has been addressed, and a specific class of perturbations have been examined. A discussion on the potential aspects of these networks in regards to new directions in algorithm design with cyclic quantum networks has also been given.

One specific new finding is a novel implementation of a quantum memory using cyclic quantum networks. A quantum memory via cyclic networks can potentially be used in experimental systems where repeating unitary operations are preferred to traditional quantum memories where information is preserved via no applications of quantum gate operations. Also, a type of quantum sensor similar to the cyclic quantum memory (modified with an acyclic perturbation line) has been given. One possible research direction for cyclic quantum sensors will be to find ways of increasing sensor capabilities with quantum algorithms.

\section{ACKNOWLEDGMENT}

We would like to thank Alberto Rojo, Ben Zeidman and Roy Clarke for helping the first author complete this work as partial fulfillment of the requirements for the Doctor of Philosophy in Applied Physics at the University of Michigan. This work was facilitated in part by a National Physical Science Consortium Fellowship and by stipend support from the National Security Agency. Additional support for this work was provided by the U.S. Department of Energy, Nuclear Physics Division, under Contract No. W-31-109-ENG-38.
[1] A. Yao, Proceedings of the 34th Annual Symposium on Foundations of Computer Science pp. 352-361 (1993).

[2] L. K. Grover, Phy. Rev. Lett. 79, 325 (1997).

[3] A. Kitaev, quant-ph/9511026.

[4] R. Cleve, A. Ekert, C. Macchiavello, and M. Mosca, Proc. R. Soc. Lond. A 454, 339 (1998).

[5] P. Benioff, Superlattices and Microstructures 23, 407 (1998).

[6] P. Benioff, Physical Review A 58, 893 (1998).

[7] S. Lloyd, Physical Review A 62, 022108/1 (2000).
[8] S. Lloyd and L. Viola, Physical Review A 65, 010101/1 (2000).

[9] H. Wiseman and G. Milburn, Physical Review A 49, 4110 (1994).

[10] A. Doherty and K. Jacobs, Physical Review A 60, 2700 (1999).

[11] M. Hamermesh, Group Theory And Its Application To Physical Problems (Dover, 1989).

[12] A. Barenco, C. H. Bennett, R. Cleve, D. P. DiVincenzo, N. Margolus, P. Shor, T. Sleator, J. A. Smolin, and 
H. Weinfurter, Physical Review A 52, 3457 (1995).

[13] D. DiVincenzo, Proc. R. Soc. Lond. A 454, 261 (1998).

[14] M. Nielsen and I. Chuang, Quantum Computation and Quantum Information (Cambridge, 2000).

[15] A. Ekert, P. Hayden, H. Inamori, and O. D.K.L., International Journal of Modern Physics A 16, 3335 (2001).

[16] M. Reck and A. Zeilinger, Phy. Rev. Lett. 73, 58 (1994).

[17] F. D. Murnaghan, The Unitary and Rotation Groups (Spartan Books, 1962).

[18] H. Goldstein, Classical Mechanics (Addison-Wesley,
1980).

[19] S. Lipschutz, Linear Algebra (McGraw-Hill, 1991).

[20] G. Birkhoff and S. Mac Lane, A Survey of Modern Algebra (Macmillan, 1965)

[21] E. Weisstein, URL http://mathworld.wolfram.com CubicEquation.html.

[22] M. McCord Nelson and W. T. Illingworth, A Practical Guide to Neural Nets (Addison-Wesley, 1991).

[23] J. J. Hopfield and D. W. Tank, Science 233, 625 (1986). 